\title{
OS EFEITOS DA ABORDAGEM FISIOTERAPÊUTICA NA QUALIDADE DE VIDA DE PACIENTES APÓS ACIDENTE VASCULAR ENCEFÁLICO: REVISÃO SISTEMÁTICA
}

\author{
THE EFFECTS OF PHYSIOTHERAPEUTIC APPROACH ON THE QUALITY \\ OF LIFE OF STROKE PATIENTS: A SYSTEMATIC REVIEW
}

\author{
Ruth Jardel Alves Alexandre Ribeiro', Charlanne de Oliveira Marques², Felipe Aurélio Nunes de Sousa ${ }^{3}$, \\ Michelle Vicente Torres ${ }^{4}$
}

${ }^{1}$ Faculdade Santo Agostinho - Teresina (PI), Brasil.

${ }^{2}$ Faculdade Santo Agostinho - Teresina (PI), Brasil.

${ }^{3}$ Faculdade Santo Agostinho - Teresina (PI), Brasil

${ }^{4}$ Universidade de São Paulo - Teresina (PI), Brasil.

\section{RESUMO}

Introdução: O acidente vascular encefálico determina um quadro neurológico. É causado pela obstrução vascular que gera isquemia e/ou extravasamento sanguíneo em determinada área encefálica. Isso pode causar déficits neurológicos graves, resultando em redução da amplitude de movimento, da força e da funcionalidade, afetando diretamente a capacidade de realização das atividades de vida diária, convívio social, situação emocional e, consequentemente, a qualidade de vida desses pacientes. Objetivo: Realizar uma revisão sistemática sobre os efeitos da abordagem fisioterapêutica na qualidade de vida desses pacientes. Materiais e Método: Foram analisados ensaios clínicos randomizados e estudos de casos de intervenção fisioterapêutica em indivíduos acometidos por acidente vascular encefálico, publicados nos idiomas inglês, espanhol e português de 2003 a 2013, disponíveis online e que retratassem terapias fisioterapêuticas na qualidade de vida desses pacientes. Foram utilizados os indexadores fisioterapia and acidente vascular encefálico and qualidade de vida, nas ferramentas de pesquisa científica Biblioteca Virtual em Saúde (BVS) e Physiotherapy Evidence Database (PEDro). Resultados: Os estudos selecionados envolveram diversas abordagens fisioterapêuticas, variando desde a educação em saúde e terapia de restrição de movimento à utilização de softwares altamente desenvolvidos que auxiliaram no processo de reabilitação, promovendo melhorias no controle e uso do membro hemiparético, nas atividades de vida diária, na satisfação pessoal, na mobilidade funcional, marcha, função cardiorrespiratória e independência desses pacientes, assim como na redução da dor. Conclusão: A fisioterapia atua diretamente no ganho de aspectos físicos, funcionais, sociais e emocionais para esses pacientes, contribuindo de forma eficaz para sua qualidade de vida.

Palavras-chave: fisioterapia; acidente vascular cerebral; qualidade de vida.

\section{ABSTRACT}

Introduction: Strokes set a neurological condition caused by a vascular obstruction, leading to ischemia and/or blood leakage in a certain brain area. They can cause severe neurological deficits resulting in reduced range of motion, less strength and less functionality. All this affects directly the patient's ability to perform daily activities, social interaction, emotional situation and, consequently, the life quality of those patients. Objective: To conduct a systematic review of the effects of physiotherapeutic approach in the quality of life of stroke patients. Materials and Methods: Random clinical trials and case studies on physical therapy intervention in individuals affected by strokes were analyzed. Those studies were published in English, Spanish and Portuguese, from 2003 to 2013, all available online. The following indexers were used: physiotherapy and stroke and quality of life. The scientific research tools used were: Biblioteca Virtual em Saúde (BVS) and Physiotherapy Evidence Database (PEDro). Results: The selected studies involved different physiotherapeutic approaches, from health education and restricted motion therapy to the use of highly developed software, which helps in the rehabilitation process by promoting improvements on the control and use of the hemiparetic member. They also benefit daily activities, personal satisfaction, functional mobility, pace, cardiorespiratory function, and patient's autonomy, as well as reducing pain. Conclusion: Physiotherapy works directly improving physical, functional, social and emotional aspects of stroke patients and effectively contributes to their quality of life.

Keywords: physical therapy specialty; stroke; quality of life. 


\section{INTRODUÇÃO}

O acidente vascular encefálico (AVE) determina um quadro neurológico, causado pela obstrução vascular que gera isquemia em determinada área encefálica e/ou extravasamento sanguíneo pelo rompimento de vasos que irrigam determinada região, causando um evento hemorrágico' ${ }^{1}$. Nos EUA, é responsável por $20 \%$ das mortes cardiovasculares, ocupando o terceiro lugar entre as causas de morte em países desenvolvidos. No Brasil, o AVE representa a primeira causa de morte por doenças cardiovasculares².

Os sintomas dessa doença podem incluir distúrbios focais ou globais da função encefálica, como distúrbio motor, incluindo falta de coordenação, unilateral ou bilateral, distúrbio sensorial unilateral ou bilateral, afasia ou disfagia, apraxias, ataxias e déficits das funções cognitivas, resultando em graus de dependência, perda de autonomia e de qualidade de vida desses indivíduos ${ }^{3}$.

Segundo a Organização Mundial de Saúde (OMS) ${ }^{4}$, qualidade de vida é a percepção do indivíduo de sua posição na vida, contexto da cultura e do sistema de valores em que vive em relação aos seus objetivos. Esse conceito, quando ligado à definição de saúde, envolve fatores biológicos, educacionais, interpessoais e de satisfação com a vida, assim como a capacidade funcional, intelectual e o próprio estado de saúde ${ }^{5}$.

A execução de atividades de vida diária (AVD), tais como vestir, alimentar-se, transferências de posições, andar, sentar e alcançar objetos estão prejudicadas nesses pacientes, interferindo no desempenho de suas atividades funcionais e na qualidade de vida ${ }^{6}$.

Nos últimos anos, o tratamento fisioterapêutico tem se mostrado efetivo na redução dos sinais e sintomas em pacientes acometidos por AVE. Utilizando-se de mecanismos de adaptação, substituição, reaprendizado, exercícios de habituação, coordenação, treinamento de equilíbrio e fortalecimento pode-se promover a independência nas AVD, nas tarefas de alto nível e de cuidados pessoais, assim como contribuir para a manutenção do condicionamento físico, além de permitir um ambiente mais favorável para a integração social, psicológica e condição de vida ativa independente ${ }^{7}$.

Diante da relevância do tratamento fisioterapêutico para esses pacientes, o objetivo deste estudo é analisar através de uma revisão sistemática os efeitos da abordagem fisioterapêutica na qualidade de vida de pessoas acometidas por AVE.

\section{METODOLOGIA}

Este estudo se caracteriza como uma revisão sistemática sobre os efeitos da abordagem fisioterapêutica na qualidade de vida de pacientes acometidos por AVE. Foram utilizadas as bases de dados eletrônicas MEDLINE, LILACS e SciELO, através das ferramentas eletrônicas: Biblioteca Virtual em Saúde (BVS) e Physiotherapy Evidence Database (PEDro), utilizando-se conjuntamente os indexadores: fisioterapia and acidente vascular cerebral and qualidade de vida e physical therapy and quality of life and stroke.

Os artigos foram triados pelos descritores citados e, a partir de um número máximo de artigos, os seguintes critérios foram sendo aplicados para refinar a pesquisa: textos completos disponíveis; assuntos principais como sendo AVE, modalidades de fisioterapia e qualidade de vida.

Foram incluídos estudos randomizados controlados e estudos de caso disponíveis, publicados no período de 2003 a 2013, nos idiomas português, espanhol e inglês, que retratassem abordagens fisioterapêuticas no tratamento de pacientes acometidos por AVE e que avaliassem a qualidade de vida desses pacientes.

Foram excluídos artigos de revisão sistemática e de literatura, teses e dissertações, assim como artigos cujo texto completo não fosse disponível nem mesmo após busca em biblioteca de referência, ou seja, aqueles cuja aquisição, por algum problema técnico, não fosse possível.

Os estudos selecionados foram avaliados individualmente pelos pesquisadores. Posteriormente, as considerações das análises de cada um foram sintetizados e tabulados. Cada artigo foi submetido a avaliação qualitativa e quantitativa considerando a qualidade da amostra, o tipo de intervenção realizada e seu possível efeito sobre a qualidade de vida, o instrumento utilizado para avaliação, os principais resultados do tratamento fisioterapêutico para o paciente e sua implicação na qualidade de vida

\section{RESULTADOS}

Foram identificados 100 estudos, mas selecionados apenas 13 , de acordo com os critérios de inclusão e exclusão, conforme o fluxograma da metodologia do estudo (Figura 1). Destes, 10 artigos foram ensaios clínicos randomizados e 3 artigos distribuídos em série de casos e estudos de caso. Os estudos variaram em relação aos objetivos e intervenções abordadas, incluindo: treinamento aeróbio, terapia por restrição do movimento do membro não parético e/ou tronco, treinamento sensório-motor, treinamento do sentar/levantar em diferentes repetições, uso de dispositivos auxiliares robóticos e games, mobilização precoce do paciente em unidade de terapia intensiva, treino isolado dos membros inferiores e 


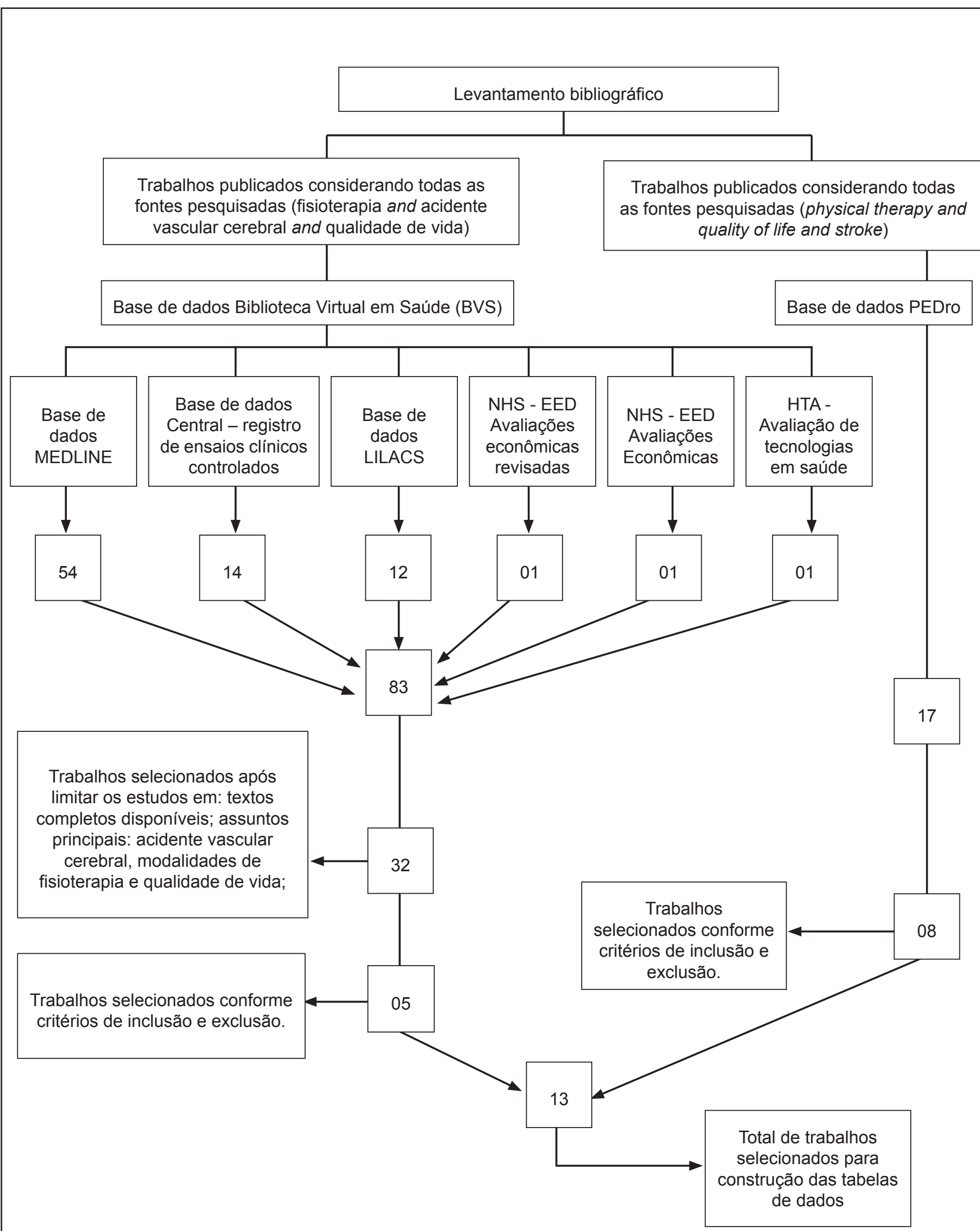

Fonte: Biblioteca Virtual em Saúde, 2013; PEDro, 2013.

Figura 1: Fluxograma de pesquisa sistemática de artigos abordando o efeito do tratamento fisioterapêutico na qualidade de vida de portadores de acidente vascular encefálico. 
superiores, uso do conceito neuroevolutivo Bobath, uso da toxina botulínica associada ao Transcutaneous electrical stimulation (TENS) e a educação em saúde. Foram avaliados: equilíbrio, força muscular, marcha, funcionalidade, dor, satisfação pessoal e realização de atividades de vida diária como pré-requisito para uma boa qualidade de vida. As características de cada estudo são verificadas no Quadro 1.

Quadro 1: Estudos que avaliaram a qualidade de vida de pessoas acometidas por acidente vascular encefálico após tratamento fisioterapêutico.

\begin{tabular}{|c|c|c|c|c|}
\hline Autor/ano & Amostra & Instrumentos & Intervenção & Principais resultados \\
\hline Tyedin et al. ${ }^{8}$ & $\begin{array}{l}71 \text { pacientes } \\
\text { admitidos } 24 \mathrm{~h} \text { na } \\
\text { unidade de terapia } \\
\text { intensiva }\end{array}$ & $\begin{array}{l}\text { Assessment of } \\
\text { Quality of Life } \\
\text { (AQoL) }\end{array}$ & $\begin{array}{c}\text { Grupo Controle: tratamento } \\
\text { convencional; } \\
\text { Grupo Intervenção: } \\
\text { mobilização precoce dentro das } \\
\text { 24h de AVC. } \\
\text { - 4x/dia até alta hospitalar }\end{array}$ & $\begin{array}{l}\text { Grupo Intervenção } \\
\text { apresentou maior qualidade } \\
\text { de vida do que o Grupo } \\
\text { Controle }\end{array}$ \\
\hline $\begin{array}{l}\text { Langhammer e } \\
\text { Stanghelle }^{9}\end{array}$ & $\begin{array}{l}61 \text { voluntários pós } \\
\text { AVE }\end{array}$ & $\begin{array}{l}\text { Nottingham Health } \\
\text { Profile (NHP) }\end{array}$ & $\begin{array}{l}\text { Fisioterapia na internação hospitalar } \\
\text { pós AVE. Grupo de Programa de } \\
\text { Reaprendizagem motora e Grupo } \\
\text { Bobath }\end{array}$ & $\begin{array}{c}\text { Redução da qualidade } \\
\text { de vida após intervenção, } \\
\text { avaliada um e quatro anos } \\
\text { após o AVE }\end{array}$ \\
\hline Duncan et al. ${ }^{10}$ & 408 voluntários & $\begin{array}{l}\text { Stroke Impact } \\
\text { Scale (SIS) }\end{array}$ & $\begin{array}{l}\text { G1: treinamento em esteira } 2 \text { meses } \\
\text { após AVE; G2: treinamento } 6 \text { meses } \\
\text { após AVE; G3: treinamento domiciliar } \\
\text { supervisionado } 2 \text { meses após AVE }\end{array}$ & $\begin{array}{c}\text { Todos os grupos } \\
\text { aumentaram a capacidade } \\
\text { funcional de caminhada e } \\
\text { qualidade de vida } \\
\end{array}$ \\
\hline Globas et al. ${ }^{11}$ & $\begin{array}{l}\text { Pessoas idosas } \\
\text { hemiparéticas e mais } \\
\text { de } 6 \text { meses de AVE }\end{array}$ & $\begin{array}{c}\text { Short- } \\
\text { Form } 12(\mathrm{SF}-12)\end{array}$ & $\begin{array}{c}3 \text { meses de intervenção. } \\
\text { Grupo de treinamento em esteira } \\
\text { (TAEX) e } \\
\text { Grupo Controle (fisioterapia } \\
\text { convencional) }\end{array}$ & $\begin{array}{c}\text { TAEX aumentou } \\
\text { significativamente a } \\
\text { mobilidade funcional da } \\
\text { marcha e escores mentais } \\
\text { do SF-12 } \\
\end{array}$ \\
\hline Barreca et al. ${ }^{12}$ & 48 voluntários & $\begin{array}{l}\text { Linkert Scale } \\
\text { Escala Visual } \\
\text { Analógica }\end{array}$ & $\begin{array}{c}\text { Grupo Controle: terapia } \\
\text { convencional; } \\
\text { Grupo Intervenção: treino do sentar/ } \\
\text { levantar }\end{array}$ & $\begin{array}{l}\text { Satisfação, independência } \\
\text { e qualidade de vida } \\
\text { foram maiores no Grupo } \\
\text { Intervenção }\end{array}$ \\
\hline McClellan e Ada ${ }^{13}$ & $\begin{array}{l}26 \text { pacientes, após } \\
18 \text { meses de AVE }\end{array}$ & $\begin{array}{c}\text { Sickness } \\
\text { Impact } \\
\text { Profile (SA- SIP30) }\end{array}$ & $\begin{array}{c}\text { G1: treino da mobilidade membro } \\
\text { inferior; G2: } \\
\text { treino da mobilidade membro } \\
\text { superior }\end{array}$ & $\begin{array}{c}\text { Programas domiciliares são } \\
\text { eficazes na qualidade de } \\
\text { vida }\end{array}$ \\
\hline Soares et al. ${ }^{14}$ & $\begin{array}{l}6 \text { pacientes com } \\
6 \text { meses de lesão }\end{array}$ & $\begin{array}{l}\text { Perfil de Saúde de } \\
\text { Nottingham }\end{array}$ & Biofeedback em plataforma instável & $\begin{array}{l}\text { Melhora do equilíbrio, } \\
\text { qualidade de vida e } \\
\text { satisfação pessoal } \\
\end{array}$ \\
\hline Moreira et al. ${ }^{15}$ & $\begin{array}{l}1 \text { paciente do sexo } \\
\text { feminino }\end{array}$ & $\begin{array}{l}\text { Escala Analógica } \\
\text { Visual }\end{array}$ & $\begin{array}{c}\text { Toxina botulínica A associada à } \\
\text { Fisioterapia }\end{array}$ & $\begin{array}{c}\text { Redução da dor; melhora } \\
\text { das atividades da vida diária } \\
\text { e qualidade de vida }\end{array}$ \\
\hline Wu et al. ${ }^{17}$ & $\begin{array}{c}26 \text { pacientes idosos } \\
\text { pós AVE }\end{array}$ & $\begin{array}{l}\text { Stroke Impact } \\
\text { Scale (SIS) }\end{array}$ & $\begin{array}{l}\text { Grupo Intervenção: terapia de } \\
\text { restrição do movimento; Grupo } \\
\text { Controle: terapia convencional }\end{array}$ & $\begin{array}{c}\text { Melhora mais significativa } \\
\text { do Grupo Intervenção na } \\
\text { função motora e qualidade } \\
\text { de vida } \\
\end{array}$ \\
\hline Wu et al. ${ }^{18}$ & $\begin{array}{l}57 \text { pessoas com } 6 \text { a } \\
55 \text { meses pós AVE }\end{array}$ & $\begin{array}{l}\text { Stroke Impact } \\
\text { Scale (SIS) }\end{array}$ & $\begin{array}{l}\text { G1: restrição do movimento do } \\
\text { membro superior; G2: restrição } \\
\text { do tronco e membro; G3: terapia } \\
\text { convencional }\end{array}$ & $\begin{array}{l}\text { Não houveram diferenças } \\
\text { significativas entre os } \\
\text { grupos quanto à qualidade } \\
\text { de vida }\end{array}$ \\
\hline Kutner et al. ${ }^{19}$ & $\begin{array}{l}17 \text { pacientes com } 3 \text { a } \\
9 \text { meses após AVE }\end{array}$ & $\begin{array}{l}\text { Stroke Impact } \\
\text { Scale (SIS) }\end{array}$ & $\begin{array}{c}\text { Treino do membro parético. Grupo } \\
\text { Controle de Terapia Convencional } \\
\text { versus Grupo Intervenção com uso } \\
\text { de dispositivo robótico }\end{array}$ & $\begin{array}{l}\text { Todos os grupos tiveram } \\
\text { significativas melhoras na } \\
\text { qualidade de vida }\end{array}$ \\
\hline Schuck et al. ${ }^{20}$ & $\begin{array}{l}\text { Dois hemiplégicos } \\
\text { crônicos }\end{array}$ & $\begin{array}{l}\text { Stroke Impact } \\
\text { Scale (SIS) }\end{array}$ & Terapia com Game TX & $\begin{array}{c}\text { Aumento da qualidade de } \\
\text { vida e maior estímulo ao } \\
\text { uso do braço afetado }\end{array}$ \\
\hline Holmgren et al. ${ }^{21}$ & $\begin{array}{l}34 \text { participantes após } \\
3 \text { a } 6 \text { meses de AVE }\end{array}$ & $\begin{array}{l}\text { Short Form } \\
\text { (SF-36) }\end{array}$ & $\begin{array}{l}\text { Grupo Intervenção: exercícios de alta } \\
\text { intensidade (5 semanas) e educação } \\
\text { em saúde; Grupo Controle: apenas } \\
\text { educação em saúde }\end{array}$ & $\begin{array}{l}\text { Melhora significativa do } \\
\text { componente físico do SF-36 } \\
\text { em ambos os grupos }\end{array}$ \\
\hline
\end{tabular}

AVE: acidente vascular encefálico. 


\section{DISCUSSÃO}

Os resultados desta revisão sugerem uma escassez de artigos abordando o tema proposto, evidenciando que esta avaliação é negligenciada muitas vezes pelos pesquisadores. Esse fato representa uma limitação dos objetivos do tratamento fisioterapêutico, que deveria incluir sobretudo a recuperação da qualidade de vida do paciente, uma vez que essa intervenção necessariamente deve ter como objetivo primordial a recuperação da sua capacidade funcional.

Evidências têm mostrado que quanto mais precocemente pacientes vítimas de AVE são tratados, mais eles vivem de forma independente. Desse modo, a mobilização precoce envolvendo o treino de sentar fora do leito, o ficar de pé e leves treinos de marcha dentro da unidade de terapia intensiva nas primeiras 24 horas após o AVE tem demonstrado importantes melhorias na qualidade de vida, motivação e satisfação pessoal destes pacientes ${ }^{8}$. Entretanto, além da mobilização precoce, é fundamental o acompanhamento fisioterapêutico em longo prazo, pois se tem verificado aumento da mortalidade, assim como redução da função motora, do controle postural e das atividades da vida diária quando esses pacientes são reavaliados depois de um e quatro anos do tratamento ${ }^{9}$.

Após as disfunções cerebrovasculares, a marcha e sua velocidade constituem um dos mais determinantes e incapacitantes fatores, sendo um preditor de deficiência e riscos a outras doenças ${ }^{10}$. A marcha hemiparética produz uma redução da mobilidade, conduzindo a um ciclo vicioso de agravamento da deficiência com consequente diminuição na função cardiorrespiratória e qualidade de vida. Desse modo, o tratamento fisioterapêutico também deve abordar a reabilitação cardiorrespiratória. Comparando-se o efeito do treinamento aeróbio com esteira versus o tratamento convencional na velocidade da marcha e seu impacto na qualidade de vida de pacientes após AVE, pode-se observar melhoras significativas na velocidade máxima da marcha de $0,12 \pm 0,12 \mathrm{~m} / \mathrm{s}$ contra $0,02 \pm 0,02 \mathrm{~m} / \mathrm{s}$ no grupo controle $(p<0,001)$, assim como no consumo máximo de oxigênio, no bem estar psicológico e satisfação pessoal dos mesmos ${ }^{11}$.

Associado ao treino de marcha, a reabilitação deve conter o treinamento sequenciado da transferência de peso e do "sentar-levantar" em diferentes alturas para diminuição dos riscos de quedas, melhoria da locomoção, controle postural, independência e qualidade de vida ${ }^{12}$.

Após a reabilitação em um centro especializado, faz-se necessária a sua continuidade no ambiente domiciliar, onde o profissional de Fisioterapia deve ser criterioso na escolha da melhor conduta quando o mesmo objetiva melhorar a qualidade de vida nesses pacientes. Para McClellan e $\mathrm{Ada}^{13}$, exercícios terapêuticos isolados do membro superior ou inferior em domicílio poderiam apresentar eficácia na qualidade de vida e mobilidade dinâmica desses pacientes. Entretanto, em seu estudo, isso não se constatou, visto que sua amostra era reduzida e os exercícios eram focados somente no ortostatismo estático, fato esse que não foi suficiente para melhorar a qualidade de vida dos pacientes, visto que o mais funcional seria também o treino da deambulação e o treino de atividades funcionais.

$\mathrm{Na}$ hemiparesia, quadro clínico mais comum resultante do AVE, o déficit do controle postural é um comprometimento importante, com graves repercussões na funcionalidade desses pacientes. Ele envolve o controle da posição do corpo e seu equilíbrio no espaço para estabilidade e orientação de seus segmentos durante uma determinada tarefa. A biorretroalimentação ou biofeedback é um recurso eficaz na restauração do equilíbrio e qualidade de vida, uma vez que esse recurso pode auxiliar o paciente a retomar sua capacidade funcional. No estudo de Soares et al. ${ }^{14}$, foram analisados os efeitos terapêuticos desse recurso associado a uma plataforma "instável" no equilíbrio, postura e qualidade de vida de pacientes após AVE. Foram observadas melhoras do equilíbrio estático e dinâmico, mobilidade e alcance funcional, assim como uma média de $18,3 \%$ de comprometimento de qualidade de vida pré-intervenção que se reduziu para $13 \%$.

Síndromes dolorosas, especialmente no ombro parético, também são fatores incapacitantes da mobilidade articular e limitantes da qualidade de vida após o AVE. Nesses casos, pode ser usada a TENS associada à toxina botulínica $\mathrm{A}$ e à fisioterapia motora, onde se observa redução significativa do quadro álgico, permitindo um aumento da amplitude de movimento articular e da motricidade ativa e passiva, promovendo, assim, uma reintegração do paciente na execução das atividades de vida diária, com consequente aumento da sua qualidade de vida ${ }^{15}$.

Um tratamento fisioterapêutico constantemente observado no treino das atividades de vida diária é a terapia por restrição ao movimento. Essa terapia possui seu foco principal na recuperação do 'aprendizado do não uso', por meio da contenção do membro não parético e/ou do tronco do paciente durante a realização e treino dessas atividades, diminuindo as compensações realizadas durante o movimento ${ }^{16}$. Dessa forma, podendo ou não tornar-se uma abordagem efetiva na recuperação e qualidade de vida desses pacientes.

A contenção do membro não parético, quando comparada à terapia convencional, que aborda o membro saudável auxiliando o membro parético, pode fornecer melhoras significativas na força e uso do membro superior parético nas AVD com associada independência e melhora na qualidade. Isso se justifica, pois essa terapia promove a reorganização cortical quanto ao aprendizado do uso desse membro. Entretanto, para que essas conquistas sejam efetivas faz-se necessário o uso de programas intensos e prolongados ${ }^{17}$. 
Simultaneamente a esse tipo de contenção, estudos têm demonstrado a associação da contenção do membro saudável à contenção do tronco com órteses que fixam este à cadeira, restringindo sua porção anterior e seus movimentos rotacionais ${ }^{18}$. Em estudo realizado por Wu et al. ${ }^{18}$, que comparou a terapia de contenção do membro superior com a terapia de restrição do tronco e do membro, observou-se melhora da força da mão no grupo com restrição do tronco. Entretanto, não houve diferença nas atividades de vida diária e funcionalidade da mão, domínios analisados na qualidade de vida, provavelmente pelo grau de dificuldade de restrição ao movimento que esses pacientes tiveram durante a contenção do tronco e membro, assim como pelo próprio conceito global de qualidade vida que contempla não apenas a melhora dos movimentos da mão e aumento da força muscular, mas aspectos físicos, funcionais, sociais e emocionais, sugerindo, como citado anteriormente, a necessidade da implementação de abordagens fisioterapêuticas mais prolongadas para resultados mais efetivos.

Atualmente, as inovações tecnológicas têm fornecido suporte para a reabilitação fisioterapêutica em pacientes neurológicos, prometendo melhorias na percepção de saúde e aprendizado destes na medida em que fornecem um feedback proprioceptivo e reaprendizagem motora. Em estudo recente ${ }^{19}$ foi desenvolvido um dispositivo robótico sofisticado que se estendia do punho aos dedos, onde seu eixo de rotação, ao localizar-se na articulação do punho, resistia à flexão dessa articulação e promovia assistência a movimentos de extensão, funcionando como um dispositivo proprioceptivo. Foi observado que, após os dois meses de protocolo, os grupos que receberam o dispositivo associado ao treino de função do membro com o terapeuta, assim como o grupo que recebeu exclusivamente treinamento convencional, apresentaram melhoras clínicas significativas no humor, função motora, força da mão e aspectos sociais. Constatando-se, além da importância desses dispositivos, a necessidade da presença do terapeuta nesses protocolos, seja regulando esses sistemas, seja de forma direta na promoção da qualidade de vida desses pacientes.
A realidade virtual através de jogos interativos também tem permitido eficácia na terapia, pois oferece um método não invasivo, motivador, que integra um programa de reabilitação por repetição e propriocepção, estimulando o uso do membro superior afetado em atividades como vestir-se, cortar alimentos, beber e tomar banho e melhorando a autopercepção e qualidade de vida ${ }^{20}$.

A educação em saúde com grupos de discussão que abordem temas sobre as dificuldades, riscos de queda e sequelas deve ser incluída nos protocolos de reabilitação ${ }^{21}$, principalmente quando se busca uma melhora de qualidade de vida, prevenção, segurança e saúde mental para esses pacientes. Obtendo-se um nível mais alto de informação, estes poderão sentir-se empoderados para desenvolver fatores promotores de hábitos de vida mais saudável. Dessa forma, a intervenção educativa incluída na reabilitação poderá ser um fator que influencia na obtenção de uma melhor qualidade de vida para o portador de AVE.

\section{CONSIDERAÇÕES FINAIS}

Constata-se a eficácia das diversas abordagens fisioterapêuticas na qualidade de vida de pacientes após AVE, promovendo melhorias no controle e uso do membro hemiparético nas atividades de vida diária, na satisfação pessoal, redução da dor, mobilidade funcional, função cardiorrespiratória e independência desses pacientes. Faz-se necessário ressaltar a necessidade da assistência precoce e prolongada, assim como a continuidade desta em ambientes domiciliares e grupos de discussão em saúde, para que os ganhos adquiridos por esses pacientes assumam toda a globalidade de conceitos que se insere na definição de qualidade de vida. Entretanto, diante da quantidade escassa de estudos publicados, sugere-se a realização de mais pesquisas que incluam essa importante avaliação, para que se tenha mais evidências científicas da eficácia das técnicas de reabilitação utilizadas pelo profissional fisioterapeuta.

\section{REFERÊNCIAS}

1. Tavares TM. Acidente vascular encefálico em adultos jovens: revisão da literatura. [trabalho de conclusão de curso]. Goiânia: Especialização em Fisioterapia Neurológica, Pontificia Universidade Católica de Goiânia; 2011.

2. Castro JAB, Epstein MG, Sabino GB, Nogueira GLO, Blankenburg C, Staszko KF, Anibal Filho W. Estudo dos principais fatores de risco para acidente vascular encefálico. Rev Soc Bras Clin Med. 2009 mai-jun; 7(3):171-3.
3. Leite HR, Nunes APN, Correa CL. Perfil epidemiológico e qualidade de vida dos pacientes acometidos por acidente vascular encefálico. Arq Ciênc Saúde UNIPAR. 2011; 15(1):15-21.

4. Doi KY, Turchiari MA, Stopiglia M. O impacto do acidente vascular encefálico na qualidade de vida dos pacientes acompanhados em uma única clínica de fisioterapia universitária. Rev Inst Ciênc Saúde. 2007; 25(1):23-8. 


\section{REFERÊNCIAS}

5. Vecchia RD, Ruiz T, Bocchi SCM, Corrente JE. Qualidade de vida na terceira idade: um conceito subjetivo. Rev Bras Epidemiol. 2005 set; 8(3):246-52.

6. Cesário CMM, Penasso P, Oliveira APR. Impacto da disfunção motora na qualidade de vida em pacientes com acidente vascular encefálico. Rev Neurocienc. 2006 janmar; 14(1):6-9.

7. Piropo TGN, Durães MA, Silva LWS, Alencar MS Estilo de vida de pessoas idosas pós-acidente vascular encefálico e sua relação com a assistência de fisioterapia em domicílio. Rev Kairós. 2010; 13(2):41-56

8. Tyedin K, Cumming TB, Bernhardt J. Quality of life: an important outcome measure in a trial of very early mobilisation after stroke. Disabil Rehabil. 2010; 32(11):875-84.

9. Langhammer B, Stanghelle JK. Bobath or motor relearning programme? A follow-up one and four years post stroke. Clin Rehabil. 2003 nov; 17(7):731-4.

10. Duncan PW, Sullivan KJ, Behrman AL, Azen SP, Wu SS, Nadeau SE, Dobkin BH, Rose DK, Tilson JK, Cen S, Hayden SK. Body-weight-supported treadmill rehabilitation after stroke. N Engl J Med. 2011 maio; 364(21):2026-36.

11. Globas C, Becker C, Cerny J, Lam JM, Lindemann U Forrester LW, Macko RF, Luft AR. Chronic stroke survivors benefit from high-intensity aerobic treadmill exercise: a randomized control trial. Neurorehabil Neural Repair. 2012 jan; 26(1):85-95.

12. Barreca SDPT, Sigouin CS, Lambert C, Ansley B. Effects of extra training on the ability of stroke survivors to perform an independent sit-to-stand: a randomized controlled trial. J Geriatr Phys Ther. 2004; 27(2):59-64.

13. McClellan R, Ada L. A six-week, resource-efficient mobility program after discharge from rehabilitation improves standing in people affected by stroke: placebo-controlled, randomised trial. Aust J Physiother. 2004; 50(3):163-7.
14. Soares AV, Hochmüller ACOL, Silva P, Fronza D, Woellner SS, Noveletto F. Biorretroalimentação para treinamento do equilíbrio em hemiparéticos por acidente vascular encefálico: estudo preliminar. Fisioter Pesq. 2009 abr-jun; 16(2):132-6.

15. Moreira KLAF, Torres CKD, Dantas MDF, Andrade SMMS. Toxina botulínica A e intervenção fisioterapêutica no tratamento do ombro doloroso pós acidente vascular cerebral: relato de caso. Semina. 2007; 28(2):119-26.

16. Oliveira R. Avaliação e treinamento de alcance com restrição de tronco em pacientes hemiparéticos pós acidente vascular cerebral. [dissertação]. Campinas: Doutorado em Ciências Médicas, Universidade Estadual de Campinas; 2008.

17. Wu CY, Chen CL, Tsai WC, Lin KC, Chou SH. A randomized controlled trial of modified constraint-induced movement therapy for elderly stroke survivors: changes in motor impairment, daily functioning, and quality of life. Arch Phys Med Rehabil. 2007 mar; 88(3):273-8.

18. Wu CY, Chen YA, Lin KC, Chao CP, Chen YT. Constraintinduced therapy with trunk restraint for improving functional outcomes and trunk-arm control after stroke: a randomized controlled trial. Phys Ther. 2012 abr; 92(4):483-92.

19. Kutner NG, Zhang R, Butler AJ, Wolf SL, Alberts JL. Quality of life change associated with robotic assisted therapy to improve hand motor function in patients with subacute stroke: a randomized clinical trial. Phys Ther. 2010 abr; 90(4):493-504.

20. Schuck SO, Whetstone A, Hill V, Levine P, Page SJ. Game-based, portable, upper extremity rehabilitation in chronic stroke. Top Stroke Rehabil. 2011 nov-dez;18(6):720-7.

21. Holmgren E, Gosman-Hedström G, Lindström B, Wester $\mathrm{P}$. What is the benefit of a high-intensive exercise program on health-related quality of life and depression after stroke? A randomized controlled trial. Adv Physiother. 2010 set; $12(3): 125-33$.

\section{Endereços para correspondência:}

Ruth Jardel Alves Alexandre Ribeiro

ruthjardel@hotmail.com

Charlanne de Oliveira Marques

charlanneoliveira@hotmail.com
Felipe Aurélio Nunes de Sousa

felipeaurelio71@hotmail.com

Michelle Vicente Torres

michellevicento@hotmail.com 